\title{
Titrages de virulence du vaccin anticharbonneux sur souris Résultats expérimentaux et application pratique
}

\author{
par J. J. RIBOT, J. GILIBERT \\ avec la collaboration technique de J. A. ANDRIANAVOLONA
}

\begin{abstract}
RÉSUMÉ
Une nouvelle méthode de titrage de vaccin anticharbonneux est exposée.

Le titrage est effectué sur souris. Quinze sourıs por dilution sont utilisées, pour tester la virulence de Spores diluées au $1 / 15$ ef au 1/20 . La moyenne du temps de mort des 10 premıères souris sert à calculer la virulence de la sérıe vaccinale.

Une série de référence déjà éprauvée esl prise comme étalon, pour calculer la dilution finale du vaccin.

La moyenne du temps de mort des souris, avec la série de référence est de 45,3 heures. Une série à tester aura une virulence équivalente à la série de référence, si la moyenne des temps de mort est intérieure à l'intervalle 45,3 \pm 20,9 . Elle aura une virulence différente si la moyenne est extérieure à cet intervalle.

Dans ce cas la dilution d'emploi du vaccin est calculée par une simple règle de trois.
\end{abstract}

\section{1. - INTRODUCTION}

Depuis 1937 (1) les bovins et ovins sont immunisés annuellement à Madagascar contre le charbon bactéridien au moyen de spores de bocillus anthracis de souche atténuée, diluées en solution gélosée et alunée.

Une injection de $1 / 4$ de $\mathrm{ml}$ en sous-cutanée à la queue procure une immunité précoce, solide et relativement durable (5). La souche employée, dite A. 43, a pratiquement la même virulence que celle du premier vaccın Pasteur (3). Cette souche est conservée sous forme de spores diluées en eau physiologique glycérinée.

Les spores sont obtenues par culture de la souche A. 43 sur gélose en boîte de Roux, après une incubation de 8 jours à 370 et 8 jours à la température du Laboratoire.

Après vérification d'une bonne sporulation, on récolte dans de l'eau physiologique (25 cc par boîte de Roux) à laquelle on ajoute $25 \mathrm{cc}$ de glycérine dès que la récolte est effectuée.

Les spores en suspension dans l'eau physiologique glycérinée, sont agitées et réparties en flacons de 125 cc. Elles serviront ensuite par dilution dans une solution gélosée alunée après vérufication (inoculation à des animaux d'expérience) à préparer le vaccın.

Les boîtes ensemencées et récoltées à la même date constituent une série vaccinale. Chaque série est vérifiée séparément.

La vérification se fait un mois ou plus après la récolte.

En vue de cette vérification, les spores sont diluées dans une solution gélosée alunée, la dilution employée pour le vaccin est celle qui tue le cobaye de $300 \mathrm{~g}$ à $350 \mathrm{~g}$ en $72 \mathrm{~h}$ environ d̀ la dose de $1 / 4 \mathrm{de} \mathrm{ml}$ en sous-cutanée, un ou 2 cobayes sont utilisés par dilution. Malheureusement, très souvent les résultats de la vérification sur 
cobaye sont aberrants, un cobaye meurt en $60 \mathrm{~h}$ le $2^{\mathrm{e}}$ en $80 \mathrm{~h}$, très souvent la dilution au $1 / 20$ tue plus vite que la dilution au 1/15, parfois un cobaye ne meurt pas.

\section{2. - OBJET DE LA RECHERCHE}

L'objet du présent travall a été de trouver une méthode de vérification, permettant d'éliminer dans la mesure du possible ces résultats aberrants. L'augmentation du nombre des animaux en expérience doit en principe, constituer une méthode plus valable. Le cobaye est assez cher, I) ne se reproduit pas très vite, la souris par contre se multıplie rapidement, son prix de revient n'est pas très élevé, aussı est-ce cet animal quı a été chorsi pour vérifier la virulence des spores vaccinales. Compter les spores entrant dans le vaccin pourrait constituer une méthode de vérification, mais, d'une série à l'autre, la virulence peut varier, car la durée de séjour des spores en eau glycérinée, avant vérification varie de 1 à plusieurs mois. En effet le vaccin est préparé à la demande et une réserve de spores est toujours gardée pour farre face à une commande imprévue. La vérification se fait, d'une façon générale, peu de temps avant la fabrication du vaccin. Le vaccin livré à l'utilisateur se trouvera être en fait la dilution qui présentera les caractères de virulence signalés plus haut (autrefors, mort du cobaye en $72 \mathrm{~h}$ environ, et maintenant étude $\mathrm{du}$ temps de mort des souris). En effet l'expérimentatıon sur animal semble le meilleur procédé de vérification, qui n'exclut d'ailleurs pas la numération des spores.

La souche A 43, à partir de laquelle on obtient les spores, est, comme déjà dit, conservée en eau physiologique glycérinée. Chaque année sa virulence est vérifiée. Le bovillon de $24 \mathrm{~h}$ à la dose de $1 / 2 \mathrm{ml}$ en ID et $\mathrm{S}$. C. ne doit pas tuer le lapın. par contre $1 / 4 \mathrm{ml}$ de la culture de $24 \mathrm{~h}$ en ID ou SC dait tuer le cobaye entre 56 et $76 \mathrm{~h}$. Si la virulence a varié on peut augmenter par passage sur animal, ou la diminuer par repiquage sur gélose pauvre.

Cette virulence propre de la souche obtenue par des germes en bouilion, est à différencier de la virulence des spores récoltées en eau physiologique glycérinée. Ce sont les spores qui serviront à fabriquer, par dilution en solution gélosée alunée, un vaccın, qui doit présenter lul-même ainsi que nous l'avons expliqué plus haut un certain caractère de virulence, sur les animaux de laboratoıre. D'une façon générale, la dilution vaccinale se situe entre le 1/15 ef le $1 / 20$. C'est l'étude sur les souris de la virulence de la dilution vaccinale qui a été effectuée.

Afin de simplifier les manipulations une sevule solution d'alun (*) était préparée à 3 p. 100 ef la dilution de spores ainsi effectuée :

$1 / 15\left\{\begin{array}{l}2.000 \mathrm{ml} \text { solution gélosée à } 2 \mathrm{p} .100 \\ 800 \mathrm{ml} \text { solution alunée }\left(^{*}\right) \text { d̀ } 3 \mathrm{p} .100 \\ 200 \mathrm{ml} \text { spores conservées en glycérine } \\ \text { et eau physiologique } \\ 2.000 \mathrm{ml} \text { solution gélosée à } 2 \mathrm{p} .100 \\ 850 \mathrm{ml} \text { solution alunée à } 3 \mathrm{p} .100 \\ 150 \mathrm{ml} \text { spores conservées en glycérine } \\ \text { et eau physiologique. }\end{array}\right.$

Donc pour un volume total de 3 ! de solution vaccinale, 2 variables entraient en jeu :

a) le nombre de spores,

b) la quantité d'alun, qui se retrouvait être de 0,8 p. 100 pour la dilution au $1 / 15$ soit $24 \mathrm{~g}$ pour 31 et de 0,85 p. 100 pour la dilution au $1 / 20$ soit $25,5 \mathrm{~g}$.

Ce mode de dilution a permis de poser les bases d'une étude, qui sera poursuivie ultérieurement sur l'influence de la quantité d'alun et la virulence résultante (4).

C'est afin d'harmoniser les titrages, de pouvoir les multiplier sur un grand nombre d'animaux, et à la suite des travaux de Norman G. Roth, Ira A. de Armon, et David H. Lively (2) que la souris a été choisie.

La souris est d'un emploi commode pour plusieurs raisons :

1) économie ;

2) facilité de constituer des lots homogènes:

3) possibilité d'emploi d'un plus grand nombre d'animaux et, de ce fait, étude statistique de la virulence :

4) suppression ou, tout au moins, diminution, sur un ensemble d'animaux, du facteur idiosyncrasique.

* Il s'agir d'alun cristallisé de formule $\left(\mathrm{SO}_{4}\right) 3 \mathrm{Al} 2$, $\mathrm{SO}_{4}$ k 2, $24 \mathrm{H}_{2} \mathrm{O}$. 


\section{3. - TRAVAUX D'APPROCHE}

a) Détermination du nombre de souris nécessaires pour un titrage valable.

Le 1 er temps de ce travail a été de déterminer le nombre moyen d'animaux nécessaires pour effectuer un titrage valable et dont les résultats seraient statistiquement exploitables.

La dose injectée est de $0,25 \mathrm{mi}$ en intra péritonéale à la souris, et de $0,25 \mathrm{ml}$ par voie souscutanée au cobaye.

Pour chaque dilution 20 souris ef 2 cobayes ont été employés et les résultats sont consignés dans le tableau ci-dessous. Bien entendu après la mort, il a été vérifié que les animaux sont morts de charbon, par recherche et coloration au gram du frottis de sang du cour, autopsie et ensemencement.

Les animaux étaient contrôlés pendant la journée toutes les heures, la nuit aussi fréquem- ment que possible au moins une fois entre $21 \mathrm{~h}$ et $6 \mathrm{~h}$ du matin (tout au moins les jours de semaine, le dimanche matın et soır, quelquefois plus.

Du tableau 1, on peut tirer comme première conséquence que :

1) dans tous les cas de 1 à 5 souris sur 20 ne meurent pas.

2) Il est possible d'établir une courbe de répartition par classes du temps de mort. L'observation de cette courbe montre que les 10 premières mortalités sont groupées, alors que les mortalıtés suivantes s'étalent d'une façon assez anarchique.

En conséquence, il a semblé rationnel pour les titrages suivants, de prendre des lots de 15 souris et de considérer les temps de mort des 10 premiers animaux. Les résultats indiqués sur le graphique I ont alors été obtenus.

\section{COURBE DE REPARTITION}

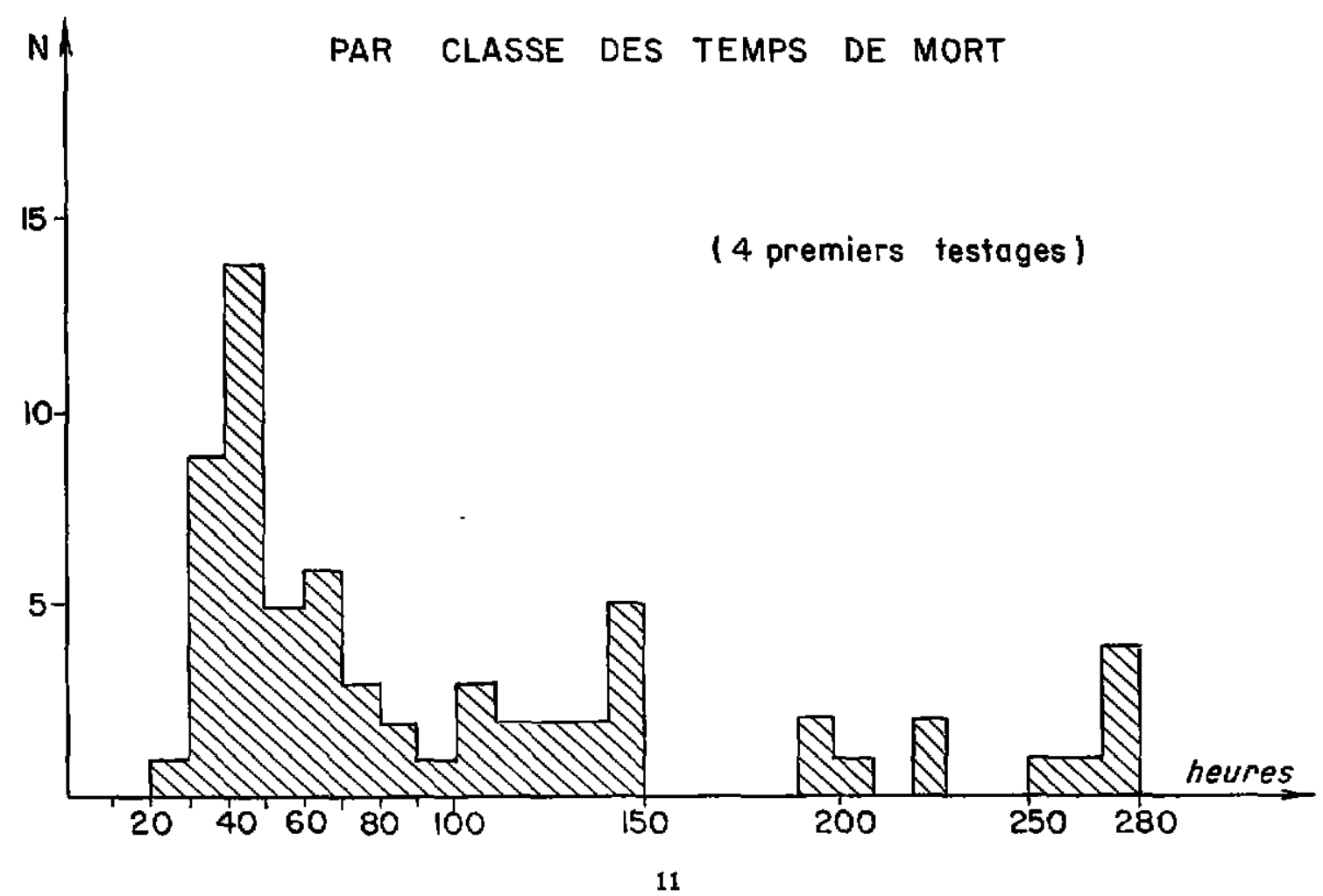


TABLEAU NO I

Détermination du nombre de souris nécessaires pour le titrage.

\begin{tabular}{|c|c|c|c|c|c|c|c|c|c|c|c|c|c|}
\hline \multirow{5}{*}{$\begin{array}{c}N^{0} \begin{array}{c}\text { de sérte } \\
\text { \& date }\end{array} \\
\text { d'Inoculat1on } \\
5 / 62 \\
23 \text { mars } \\
1964\end{array}$} & \multirow{5}{*}{ 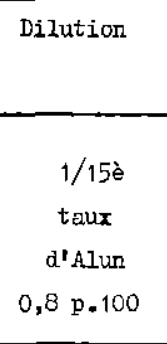 } & \multicolumn{5}{|c|}{ Souris inoculées } & \multicolumn{5}{|c|}{$\begin{array}{l}\text { Temps écoulé entre Inoculation et } \\
\text { constat de mort en heures }\end{array}$} & \multirow{5}{*}{$\begin{array}{l}\text { Cobayes } \\
\begin{array}{l}207 \mathrm{~g} \\
274 \mathrm{~g}\end{array}\end{array}$} & \multirow{5}{*}{$\begin{array}{c}\begin{array}{c}\text { Temps } \\
\text { écoulé } \\
\text { entre I\&N }\end{array} \\
68 \mathrm{H} \\
80-84 \mathrm{H}\end{array}$} \\
\hline & & \multirow{2}{*}{$\frac{1}{6}$} & \multirow{2}{*}{$\frac{2}{7}$} & \multirow{2}{*}{$\frac{3}{8}$} & \multirow{2}{*}{$\frac{4}{9}$} & 5 & 25 & \multirow{2}{*}{$\frac{33-37}{33-37}$} & \multirow{2}{*}{$\frac{33-37}{33-37}$} & \multirow{2}{*}{$\frac{33-37}{33-37}$} & \multirow{2}{*}{$\frac{33-37}{43}$} & & \\
\hline & & & & & & 10 & $33-37$ & & & & & & \\
\hline & & 11 & 12 & 13 & 14 & 15 & 43 & 43 & 46 & 47 & 52 & & \\
\hline & & 16 & 17 & 18 & 19 & 20 & 53 & 60 & 71 & 117 & & & \\
\hline \multirow{4}{*}{$\begin{array}{l}5 / 61 \\
23 \text { mars } \\
1964\end{array}$} & \multirow{4}{*}{$\begin{array}{c}1 / 20 \mathrm{e} \\
\text { taux } \\
d^{\prime} \text { Alun } \\
0,35 \text { p.100 }\end{array}$} & 1 & 2 & 3 & 4 & 5 & 43 & 43 & 43 & 46 & 46 & \multirow{4}{*}{$\begin{array}{l}235 \mathrm{~g} \\
245 \mathrm{~g}\end{array}$} & \multirow{4}{*}{$\begin{array}{r}75 \text { н } \\
283 \text { н }\end{array}$} \\
\hline & & 6 & 7 & 8 & 9 & 10 & 60 & 60 & 61 & $7 j$ & $82-35$ & & \\
\hline & & 11 & 12 & 13 & 14 & 15 & $124-133$ & $142-147$ & $142-147$ & 193 & 250 & & \\
\hline & & 16 & 17 & 18 & 19 & .20 & $266-278$ & $266-278$ & $21 \mathrm{~J}$ & & & & \\
\hline \multirow{4}{*}{$\begin{array}{l}6 / 62 \\
\text { mars } \\
1964\end{array}$} & \multirow{4}{*}{$\begin{array}{c}1 / 15 e ̀ \\
\text { talux } \\
\text { d'Alun } \\
0,8 p \cdot 100\end{array}$} & 1 & 2 & 3 & 4 & 5 & 43 & 46 & 46 & 47 & 52 & \multirow[t]{4}{*}{$295 \mathrm{~g}$} & \multirow[t]{4}{*}{$80-85 \mathrm{H}$} \\
\hline & & 6 & 7 & 8 & 9 & 10 & 53 & 60 & 60 & 120 & $124-138$ & & \\
\hline & & 11 & 12 & 13 & 14 & 15 & $202-207$ & $217-235$ & $217-235$ & 257 & $266-278$ & & \\
\hline & & 16 & 17 & 18 & 19 & 20 & $266-278$ & & & & & & \\
\hline \multirow{4}{*}{$\begin{array}{l}6 / 62 \\
\operatorname{mars} \\
1964\end{array}$} & \multirow{4}{*}{$\begin{array}{c}1 / 20 \mathrm{è} \\
\text { taux } \\
\text { d'Alum } \\
0,85 p .100\end{array}$} & 1 & 2 & 3 & 4 & 5 & $33-37$ & 46 & 46 & 52 & 73 & \multirow[t]{4}{*}{$224 g$} & \multirow[t]{2}{*}{$92 \mathrm{H}$} \\
\hline & & 6 & 7 & 8 & 9 & 10 & 96 & 100 & 107 & 107 & 113 & & \\
\hline & & 11 & 12 & 15 & 14 & 15 & $124-138$ & $142-147$ & $142-147$ & $142-147$ & 191 & & $142-152 \mathrm{E}$ \\
\hline & & 16 & 17 & 18 & 19 & 20 & & & & & & & \\
\hline
\end{tabular}


b) Influence de la concentration de I'alun. Comparaison statistique des 10 premiers temps de mort pour les 2 dilutions :

Dilution 0,80 p. $100: \bar{x}=55,7 ; \quad S m=7,46$.

Dilution 0,85 p. $100: \bar{x}=26,2 ; \quad S m=3,25$. $\dagger=2,75$, la différence entre les deux lots est significative au seui! 0,05 .

La dilution de l'alun a donc une grande importance dans la virulence.

c) Influence du poids des souris.

Dans le titrage suivant, pour une dilution au $1 / 15,2$ lots de souris ont été constitués. Un lot de 15 grosses souris de poids moyen $22 \mathrm{~g}$ et un lof de souris plus jeunes, donc moins grosses de poids moyen $14 \mathrm{~g}$, les résultats obtenus sont résumés dans le tableau suivant:

Comparaison statistique des 2 lots de souris :

Grosses souris $=\bar{x}=38,8 ； \quad S m=4,02$.

Petites souris $=\bar{x}=28,5 ; \quad S m=1,58$.

$\dagger=1,83$; la différence entre les 2 lots est donc significative au seuil de 0,10 . Le calcul montre qu'il convient d'utiliser pour les expériences, des souris de taille moyenne et homogène (poids : $20 \mathrm{~g}$ de moyenne).

Nota $=$ Chez le cobaye, sur deux animaux il est vrai, le plus gros est mort le premier.

TABLFAU NO II

Influence de la concentration de ltAlun

\begin{tabular}{|c|c|c|c|c|c|}
\hline $\begin{array}{l}\text { Série 1/63 } \\
\text { (Mai 1964) }\end{array}$ & $\begin{array}{c}1 / 15 \\
\text { taux Alun } \\
0,80 \mathrm{p} \cdot 100\end{array}$ & Souris inoculées & $\begin{array}{l}\text { Temps de mort (en H) } \\
\begin{array}{l}26-39-41-49-51- \\
51-53-67-67-113- \\
----\quad-\end{array}\end{array}$ & $\begin{array}{l}\text { cobsyes } \\
\text { inoculés } \\
355 \mathrm{~g} \\
395 \mathrm{~g}\end{array}$ & $\begin{array}{l}\text { Temps de } \\
\text { mort } \\
80 \text { H } \\
105 \text { H }\end{array}$ \\
\hline $\begin{array}{l}\text { Série 1/63 } \\
\text { (Mai 1964) }\end{array}$ & $\begin{array}{c}1 / 15 \\
\text { taux Alun } \\
0,85 \text { p. } 100\end{array}$ & 15 & $\begin{array}{l}13-18-18-19-23- \\
26-27-33-42-43- \\
66-67-72-90-96-\end{array}$ & $\begin{array}{l}251 \mathrm{~g} \\
340 \mathrm{~g}\end{array}$ & $\begin{array}{l}20 \text { В } \\
49 \text { Е }\end{array}$ \\
\hline
\end{tabular}

TABLEAU No III

Influence du poids des souris.

\begin{tabular}{|c|c|c|c|c|c|c|c|c|c|}
\hline $\begin{array}{l}\text { No Sátie } \\
\text { Date } \\
\text { Inoculation }\end{array}$ & Dilution & Souris inoculées & & $\begin{array}{l}\text { emps } \\
\text { lat: } \\
\text { mor }\end{array}$ & $\begin{array}{l}\text { coul } \\
\text { et } \\
\text { en } h\end{array}$ & $\begin{array}{l}\text { entr } \\
\text { nate } \\
\text { res) }\end{array}$ & & $\begin{array}{l}\text { Cobayes } \\
\text { inoculés }\end{array}$ & $\begin{array}{l}\text { Temps écoulé } \\
\text { entre } \\
\text { inoculation } \\
\text { et constat } \\
\text { de mort }\end{array}$ \\
\hline \multirow{3}{*}{$\begin{array}{l}\text { Série 2/63 } \\
\text { Juin } 1964\end{array}$} & \multirow{3}{*}{$\begin{array}{c}\text { taur Alun } \\
0,85 \mathrm{p} .100 \\
\text { grosses souris }\end{array}$} & \multirow{3}{*}{$\frac{15}{\text { poids moyen } 22 \text { Ex }}$} & 18 & 30 & 30 & 32 & $\begin{array}{l}32 \\
45\end{array}$ & \multirow{3}{*}{$435 \mathrm{~g}$} & \multirow{3}{*}{$77 \mathrm{H}$} \\
\hline & & & $\begin{array}{l}32 \\
45\end{array}$ & $\begin{array}{l}32 \\
45\end{array}$ & 48 & $\begin{array}{l}50 \\
67\end{array}$ & $\begin{array}{l}50 \\
67\end{array}$ & & \\
\hline & & & $\begin{array}{l}50 \\
67\end{array}$ & & & & & & \\
\hline \multirow{3}{*}{$\begin{array}{l}\text { Sárie 2/63 } \\
\text { Juin } 1964\end{array}$} & $t / 15$ & \multirow{3}{*}{ poids moyen 14 st } & 22 & 25 & 25 & 25 & 25 & \multirow{3}{*}{$396 \mathrm{~g}$} & \multirow{3}{*}{$81 \mathrm{H}$} \\
\hline & taur Alum & & 30 & 30 & 32 & 32 & $\begin{array}{l}32 \\
45\end{array}$ & & \\
\hline & petites sauris & & $\begin{array}{l}32 \\
45\end{array}$ & $\begin{array}{l}32 \\
45\end{array}$ & $\begin{array}{l}32 \\
45\end{array}$ & 71 & & & \\
\hline
\end{tabular}




\section{4. - EXPLOITATION DES RÉSULTATS}

a) Tableau des résultats, temps de mort des 10 premières souris.

Nota $=$ Quand la mort d'une souris a été constatée après un intervalle de temps de plus d'une heure, nous avons pris comme temps de mort la moyenne entre les deux derniers contrôles.

b) Recherche de l'écart-type de l'erreur pour 1 cellule.

L'analyse totale des résultats donne pour l'ensemble des 8 cetlules l'écart-type :

$$
\text { erreur }=39,75
$$

Nota $=$ Comparaison des $s_{\mathrm{e}}$ pour les dilutions $1 / 15$ et $1 / 20$.

Dilution $1 / 15$

$$
\text { se }=45,43
$$

Dilution $1 / 20$

$$
\text { se }=33,62
$$

Les $s_{e}$ pour les dilutions au $1 / 15$ ef au $1 / 20$ sont peu différents, nous aurons donc bien le droit de comparer les cellules 2 à 2 .

TABLEAU NO IV

Temps de mort des 10 premières souris.

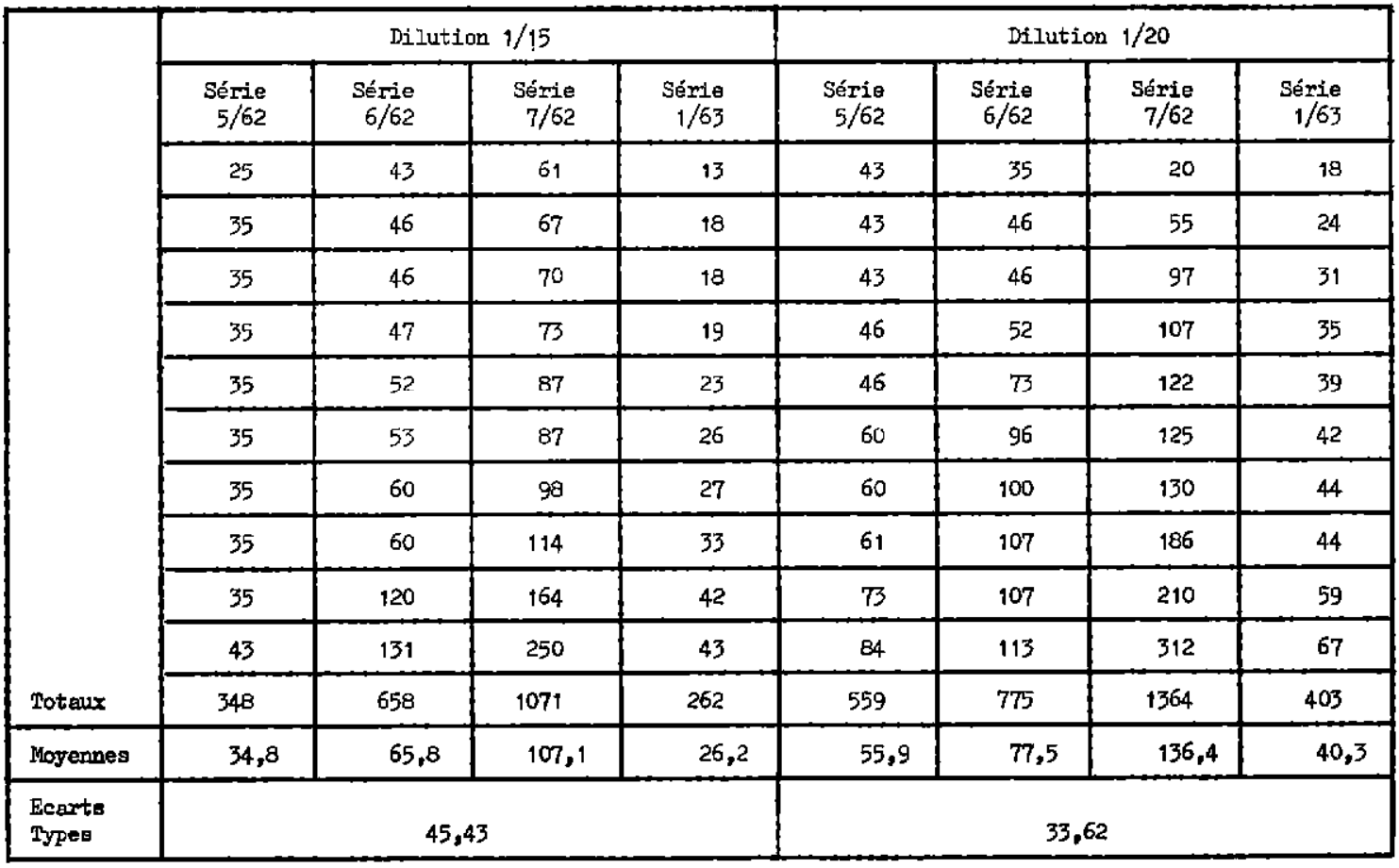

\section{5. - COMPARAISON DE DEUX SÉRIES}

a) Cas général :

Pour comparer 2 séries quelconques (série 1 et série 2) ; nous allons comparer : les moyennes $M_{1}$ et $M_{2}$ des valeurs obtenues pour chaque série avec les dilutions au 1/15 et au 1/20:

$$
M_{1}=\frac{m_{1(1 / 15)}+m_{1(1 / 20)}}{2}
$$

ef

$$
M_{2}=\frac{m_{2(1 / 15)}+m_{2(1 / 20)}}{2}
$$

La différence entre les séries (1) eł (2) sera considérée comme significative lorsque $\left|M_{1}-M_{2}\right|$ sera supérieur à $\dagger \times 5 M_{1}-M_{\text {: }}$ 
1) Valeur de $S M_{1}-M_{2}$

$$
\left.\begin{array}{c}
s_{M_{1}}^{2}=\frac{s_{e}^{2}}{4}\left(\frac{1}{10}+\frac{1}{10}\right) \\
s_{M_{1}}^{3}=\frac{s_{e}^{2}}{4}\left(\frac{1}{10}+\frac{1}{10}\right)
\end{array}\right\} \begin{gathered}
s_{M_{1}}^{2}-M_{2}=s_{M_{1}}^{2}+s_{M_{2}}^{2}=\frac{s_{e}^{2}}{10} \\
s_{M_{1}}-M_{2}=\frac{s e}{10}=\frac{39,75}{3,17} s_{M_{1}-M_{2}}=12,50
\end{gathered}
$$

2) Valeur de $\dagger$

On prend $P=0,10$, ce qui donne $t=1,67$

3) Voleur de $\dagger S_{M_{1}}-M_{2}$

$+5 M_{1}-M_{2}=1,67 \times 12,50=20,9$.

Les deux séries (1) et (2) seront considérées comme ayant des virulences différentes lorsque :

$$
\left|M_{1}-M_{2}\right|>20,9
$$

Nota. La valeur 20,9 est due d'une part à la dispersion des résultats à l'intérieur d'une colonne, d'autre part au nombre limité de séries testées (4 à ce jour); dans les années à venir, le nombre de séries testées va augmenter, la valeur de référence $(20,9)$ va diminuer, ef cette méthode de testage de virulence deviendra plus fine.

\section{b) Série de référence.}

La souche 5/62 a été choisie comme souche de référence. Elle a été effectivement employée à la dilution $1 / 15$, sur environ 200.000 bovins au mois d'avril, et à ce jour aucun cas de charbon n'a été signalé. On peut donc considérer que cette virulence prise pour référence de vaccin, a les caractères d'un bon vaccin à savoir efficacité et inocuité sur l'espèce employée.

Les moyennes des 10 premiers temps de mort pour cette série sont :

$$
\left\{\begin{array}{l}
\text { dilution } 1 / 15=34,8 \mathrm{~h} \\
\text { dilution } 1 / 20=55,9 \mathrm{~h} \\
\text { moyenne générale }=45,3 \mathrm{~h}
\end{array}\right.
$$

On considérera qu'une série à tester ( $S$ ) est de même virulence que la série de référence $(R)$ lorsque

$$
\left|M_{s}-M_{r}\right|<20,9
$$

Dans ce cas, la série (S) sera employée à la dilution $1 / 15$.
On considérera qu'une série $(S)$ a une virulence différente de $(R)$ lorsque

$$
\left|M_{s}-M_{r}\right|>20,9
$$

Elle sera alors employée à la dilution.

$$
\frac{1}{15} \times \frac{M s}{M r}
$$

c) Comparaison avec la série de référence $(5 / 62)$.

1) Série $5 / 62$ et $7 / 62$

$$
\begin{aligned}
& M 5 / 62=\frac{34,8+55,9}{2}=45,3 \mathrm{H} \\
& M 7 / 62=\frac{107,1+136,4}{2}=121,74
\end{aligned}
$$$$
M 7 / 62-M 5 / 62=76,44
$$

$76,4>20,9$ les 2 séries sont différentes.

La série $7 / 62$ est $\frac{121,7}{45,3} \neq 2,7$ fois moins virulente que la souche de référence /5/62) donc, dilution

$\frac{2,7}{15}=\frac{2}{11}$

2) Série $5 / 62$ et $6 / 62$.

m $5 / 62=45,3 \mathrm{H}$

$\mathrm{m} 6 / 62=71,6 \mathrm{H}$

$m 6 / 62=m 5 / 62=26,3 H$.

$26,3>20,9$ les 2 séries sont différentes.

La série $6 / 62$ est $\frac{71}{45}$ \# 1.5 fois moins virulente que la série de référence, donc dilution :

$$
\frac{1.5}{15}=\frac{1}{10}
$$

3) Série $5 / 62$ et $1 / 63$ :

$$
\begin{aligned}
& \text { m } 5 / 62=45,3 \mathrm{H} \\
& \mathrm{m} 1 / 63=33,2 \mathrm{H} \\
& \mathrm{m} 5 / 62=\mathrm{m} 1 / 63=12,1 \mathrm{H}
\end{aligned}
$$

$12,1<20,9$. On consıdère les deux séries comme semblables, d'où même dilution d'emploi au 1/15.

Volontairement, dans ces exemples, il n'a pas été tenu compte de la dose d'alun.

Le présent travail doit encore être poursuivi, et amélioré. Dans les prochains titrages, la dose d'alun sera harmonisée ef sera la même pour 
toutes les expériences. Pour les dilutions calculées dans les exemples précédents, le taux d'alun final a été celui de la dilution de référence soit 0,8 p. 100 pour les séries $6 / 62$ et $7 / 62$ mais par contre de 0,85 p. 100 pour la série $1 / 63$.

Le calcul de comparaison de virulence a été effectué avec les résultats obtenus avec cefte dose d'alun pour cette série.

En effet, et ceci a été volontaire, c'est une virulence que l'on peut qualifier de résultante (due à la souche ou due d̀ l'alun) qui a seule été considérée.

\section{CONCLUSION}

La méthode qui a été exposée est certainement critiquable mais sans doute moins que celle qui consistalt à prendre seulement 2 cobayes pour étudier la virulence d'une dilution. Elle semble cependant avoir l'avantage d'être moins aléatoire, d'être statistiquement plus valable, et surtout de présenter plus de sécurité car elle fait appel à un plus grand nombre d'animaux, tout en restant simple à mettre en œuvre.

En 1964 environ 1.000 .000 de doses de vaccin anticharbonneux ont été livrées après avoir été testées par cette méthade et ceci sans inconvénient jusqu'à ce jour, ce qui nous encourage à continuer dans cette voie.

Institut d'Elevage et de Médecine Vétérinaire
des Pays tropicaux
Laboratoire Central de l'Elevoge de Tananorive.

\section{SUMMARY}

\section{Assay of the virulence of the anti-anthrax vaccine in the mouse. Experimental results and practical applications.}

A new method of assaying the anti-anthrax vaccine is described.

The assay is performed in the mouse. Fifteen mince are used for each dilution in order to test the virulence of the Spores diluted 1/15 and 1/20. The average of the death rates of the first 10 mice is used to calculate the virulence of the vaccinal series.

A previously tested reference series is taken as standard so as to calculate the final dilution of the vaccine.

In the case of the reference series the average time of the death rates of the mice is 45.3 hours. A series that is being tested would be considered to have a virulence equivalent to that of the reference series if the average time of the death rate is within an interval of $45.3 \pm 20.9$. The virulence is considered to be different if the average lies outside this interval.

In this case the dilution for the practical aplication of the vaccine is calculated by a simple rule of three.

\section{RESUMEN}

\section{Dosificaciones de la virulencia de la vacuna contra el carbón sintomático sobre los ratones. Resultados experimentales y aplicación práctica.}

Se expone un nuevo método de dosificación de la vacuna contra el carbón sintomática. Se efectua la dosificación sobre ratones. Quince ratones por dilución son utilizados para testar la virulencia de esporas diluidas a 1 por $15 y$ 1 par 20. Con el termino medio del tiempo de muerte de los diez primeros ratones se calcula la virulencia de la serie vaccinal.

Se escoge como patrón una serje testigo ya experimentada para colcular la dilución final de la vacuna. El termino medio del tiempo de muerte de los ratones, con la serie testigo es de 45,3 horas. Una serie a testar tendrá una virulencia equivalente a la serie testigo, si el termino medio de los tiempos de muerte está comprendido entre el intervalo, 45,3 ㄴ 20,9. Su virulencia sera diferente si el termino medio está fuera de este intervalo.

En este caso, se calcula la dilución de la vacuna por una simple regla de tres. 


\section{BIBLIOGRAPHIE}

BUCK (G.). - Rapport Annuel du laboratoire central de l'élevage 1937. La vaccination des bovidés contre le charbon bactérien, : 9-22.

NORMAN (G.) ROTH, IRA A DE ARMON (J. R.) and DAVID (H.) LIVELY, - Survival Time as a rapid method of deiermining virulence with bacillus anfhracis J. bact. 1956, 72. 666-72.

RAMON (G.) et STAUB (A.). - Essais sur l'immunisation contre le charbon sur une nouvelle formule de vaccination charbonneuse. Bull. Acad. vét. de France 1936, 9 (7) : 375-87.
RAMON (G.). - Sur les bases essentielles de certaines méthodes modernes d'immunisation. Les principes des anatoxines et celui des facteurs adjuvants de l'immunité et leurs applications. Bull. Acad, vét. de France 1942, 15 (1) : 29-41.

RAMON (G.) et STAUB (A.). - Les nouveaux procédés de vaccination contre le charbon et contre le rouget au moyen d'une inoculation unique de virus-yaccin spécifique très atténué et additionné de substances stimulantes de l'immunité. Leur essor. Bull. Acod. vét. de France 1942, 15 : 50-4. 